\title{
Philosophiques
}

\section{La définition bolzanienne de l'analyticité logique}

\section{Edgar Morscher}

Volume 30, numéro 1, printemps 2003

Bernard Bolzano. Philosophie de la logique et théorie de la connaissance

URI : https://id.erudit.org/iderudit/007737ar

DOI : https://doi.org/10.7202/007737ar

Aller au sommaire du numéro

\section{Éditeur(s)}

Société de philosophie du Québec

ISSN

0316-2923 (imprimé)

1492-1391 (numérique)

Découvrir la revue

Citer cet article

Morscher, E. (2003). La définition bolzanienne de l'analyticité logique. Philosophiques, 30(1), 149-169. https://doi.org/10.7202/007737ar

\section{Résumé de l'article}

D'après Bolzano, une proposition est logiquement analytique si et seulement si elle est soit logiquement valide, soit logiquement non valide. Bolzano dit aussi parfois qu'une proposition est logiquement valide si et seulement si elle est et reste vraie sous toute variation simultanée et uniforme de ses parties non logiques. C'est essentiellement la même définition que donne Quine dans son article « Carnap and Logical Truth » où il attribue à ce dernier (et dans une note également à Bolzano) l'idée qu'un énoncé logiquement vrai est un énoncé au sein duquel seuls les termes logiques sont essentiels. Mais qu'en est-il des propositions et des énoncés vrais qui sont composés exclusivement de parties logiques ? Selon la définition précédente, elles s'avèreraient toutes logiquement valides ou logiquement vraies. Une proposition telle que « Il y a quelque chose » n'est toutefois manifestement pas logiquement valide selon Bolzano. La définition courante de la validité logique doit être modifiée de manière à répondre aux intuitions bolzaniennes. Dans cet article, je propose une telle modification.
Ce document est protégé par la loi sur le droit d'auteur. L'utilisation des services d’Érudit (y compris la reproduction) est assujettie à sa politique d'utilisation que vous pouvez consulter en ligne.

https://apropos.erudit.org/fr/usagers/politique-dutilisation/ 


\title{
La définition bolzanienne de l'analyticité logique
}

\author{
EDGAR MORSCHER \\ Université de Salzbourg \\ edgar.morscher@sbg.ac.at
}

\begin{abstract}
RÉSUMÉ. - D'après Bolzano, une proposition est logiquement analytique si et seulement si elle est soit logiquement valide, soit logiquement non valide. Bolzano dit aussi parfois qu'une proposition est logiquement valide si et seulement si elle est et reste vraie sous toute variation simultanée et uniforme de ses parties non logiques. C'est essentiellement la même définition que donne Quine dans son article «Carnap and Logical Truth » où il attribue à ce dernier (et dans une note également à Bolzano) l'idée qu'un énoncé logiquement vrai est un énoncé au sein duquel seuls les termes logiques sont essentiels. Mais qu'en est-il des propositions et des énoncés vrais qui sont composés exclusivement de parties logiques? Selon la définition précédente, elles s'avèreraient toutes logiquement valides ou logiquement vraies. Une proposition telle que «ll y a quelque chose» n'est toutefois manifestement pas logiquement valide selon Bolzano. La définition courante de la validité logique doit être modifiée de manière à répondre aux intuitions bolzaniennes. Dans cet article, je propose une telle modification.
\end{abstract}

ABSTRACT. - A proposition is logically analytic according to Bolzano if and only if it is either logically valid or logically non-valid. And a proposition is sometimes said to be logically valid according to Bolzano if and only if it is true and remains true under all simultaneous and uniform variations of all of its non-logical parts. Basically the same definition is provided by Quine in his paper "Carnap and Logical Truth" where he attributes to Carnap (and in a footnote also to Bolzano) the view that a logically true sentence is a true sentence which involves only logical words essentially. But, what about true propositions and sentences which are composed exclusively of logical parts? Due to the definition mentioned above, all of them will trivially turn out as logically valid or logically true. A proposition like "There is something", however, is clearly not logically valid according to Bolzano. The common definition of logical validity must be modified in order to match Bolzano's intuitions. In this paper, such a modification is presented.

\section{Introduction}

Bernard Bolzano est le premier logicien à avoir fourni, avec ses définitions de la «validité universelle logique » et de la "déductibilité logique », une explication des deux notions sémantiques fondamentales que sont, respectivement, la vérité logique et la conséquence logique. Qui plus est, ses définitions satisfont aux exigences de rigueur de la logique mathématique contemporaine. Alfred Tarski et Rudolf Carnap ont, cent ans plus tard, redéfini ces concepts sans connaître les travaux préliminaires de Bolzano - mais sans non plus échapper complètement à leur ascendant. Dans le meilleur des cas, leurs définitions ne surpassent celles de Bolzano que du point de vue formel, mais certainement pas en ce qui concerne leur teneur de sens.

PHILOSOPHIQUES 30/1 — Printemps 2003, p. 149-169 
$\mathrm{Au}$ vingtième siècle, des auteurs importants se sont servis des moyens offerts par la logique contemporaine pour reconstruire minutieusement les définitions bolzaniennes. On doit mentionner en première ligne les travaux de Heinrich Scholz et de Jan Berg. La plus grande partie de ce qui a été écrit sur le sujet depuis s'appuie directement ou indirectement sur ces travaux - et, malheureusement, il est rare que ce soit sans les diluer. Le but principal des considérations qui suivent est de combattre de telles «dilutions".

\section{2.}

Dans sa définition de l'analyticité logique, Bolzano fait un usage constitutif de la procédure de variation (ou substitution) simultanée et uniforme de représentations en soi (Vorstellungen an sich) dans une proposition en soi (Satz an sich $)^{1}$. Nous désignons la proposition qui résulte de la proposition $S$ lorsque sont substituées dans cette dernière simultanément et uniformément les représentations $v_{1}, \ldots, v_{n}$ par les représentations $w_{1}, \ldots, w_{n}$ par : « $S\left(v_{1}, \ldots, v_{n} / w_{1}, \ldots, w_{n}\right) »$.

\subsection{Six clauses relatives à la procédure de substitution}

Afin de s'assurer que la procédure de substitution est bien définie et qu'elle mène à des résultats univoques, on doit prendre certaines précautions. Malgré qu'on s'entende sur le but et l'utilité de telles précautions, leur teneur n'est pas toujours fixée de manière distincte et peut laisser plusieurs alternatives ouvertes. Dans nos rapports pratiques avec la procédure substitutionnelle, il est donc à chaque fois indispensable de préciser quelles substitutions sont admissibles et lesquelles ne le sont pas. C'est donc précisément parce que je suis en grande partie d'accord avec les déterminations qui sous-tendent en général l'interprétation courante que je désire commencer par expliciter six présuppositions relatives à mon application de la procédure substitutionnelle. Je souhaite de la sorte éviter que les divergences qui apparaîtront plus tard puissent être ramener à des différences concernant les présuppositions ou clauses relatives à la procédure de substitution. La première de ces six clauses est une restriction et exclut quelque chose, les autres indiquent tout ce que j'autorise en ce qui concerne la procédure substitutionnelle.

\section{Première clause}

Afin de garantir l'univocité de la procédure de substitution simultanée, on doit exiger que les représentations : $v_{1}, \ldots, v_{n}$, que l'on substitue soient mutuellement distinctes, tandis qu'il n'est pas nécessaire que les représentations $w_{1}, \ldots, w_{n}$ qui les remplacent le soient. Mais cette restriction demeure toute-

1. Bolzano utilise aussi simplement «Satz» et «Vorstellung» pour désigner les propositions et les représentations en soi. Dans le reste du texte nous adoptons cette version terminologique. Pour une présentation sommaire de la théorie bolzanienne de la proposition en soi, voir l'introduction de l'éditeure dans le même numéro. 
fois trop faible pour cautionner l'univocité de la procédure de substitution. On peut en faire la démonstration à l'aide de l'exemple suivant ${ }^{2}$ :

$$
\begin{aligned}
& \text { S1 }=\text { [Tout homme judicieux est judicieux }] \\
& \text { v1 }=\text { [homme judicieux }] \\
& \text { v2 }=\text { [judicieux }] \\
& \text { w1 }=\text { [renard rusé }] \\
& \text { w2 }=\text { [effronté }]
\end{aligned}
$$

Même si $\mathrm{v}_{1} \neq \mathrm{v}_{2}, \mathrm{~S}_{1}\left(\mathrm{v}_{1}, \mathrm{v}_{2} / \mathrm{w}_{1}, \mathrm{w}_{2}\right)$ n'est pas univoque. Elle a bien plutôt deux résultats possibles, à savoir [Tout renard rusé est effronté] ou [Tout homme effronté est effronté]. Pour cautionner l'univocité de la procédure de substitution simultanée $S\left(v_{1}, \ldots, v_{n} / w_{1}, \ldots, w_{n}\right)$, nous ne devons donc pas seulement exiger que $v_{1}, \ldots, v_{n}$ soient mutuellement distinctes mais bien plutôt qu'elles soient mutuellement exclusives au sens de la définition suivante («ssi » sert ici d'abréviation pour «si et seulement si ») :

Les représentations (ou propositions) en soi $l, \ldots, l_{n}$ sont mutuellement exclusives ssi aucune des représentations (ou aucune des propositions) $l_{1}, \ldots, l_{n}$ n'est la composante d'une autre.

Formellement :

Exclusives $\left(l_{1}, \ldots, l_{n}\right): \leftrightarrow \forall i \forall j\left[(1 \leq i \leq n \wedge 1 \leq j \leq n \wedge i \neq j) \rightarrow \neg \operatorname{partie}\left(l_{\mathrm{i}}, l_{\mathrm{j}}\right)\right]$

Il s'ensuit, naturellement, que les représentations $v_{1}, \ldots, v_{n}$ doivent aussi être mutuellement distinctes. Nous retenons donc (V1) :

(V1) $S\left(v_{1}, \ldots, v_{n} / w_{1}, \ldots, w_{n}\right)$ est définie pour les représentations $v_{1}, \ldots, v_{n}$ ssi $v_{1}, \ldots, v_{n}$ sont mutuellement exclusives.

\section{Deuxième clause}

On retrouve souvent dans la littérature une restriction plus étroite que (V1), à savoir la clause (V1') selon laquelle $v_{1}, \ldots, v_{n}$ doivent être mutuellement distinctes et simples. (V1) découle de cette clause, mais non l'inverse. Une représentation simple est ici définie de la manière suivante :

Une représentation en soi $v$ est simple ssi $v$ n'a aucune partie simple, c'est à dire ssi il n'y a aucune proposition et aucune représentation distincte de $v$ qui est la composante de $v$.

Formellement :

$$
\operatorname{Simple}(v) \leftrightarrow \neg \exists l(l \neq v \wedge \operatorname{Partie}(l, v))
$$

Lorsqu'il s'agit de savoir si une représentation est véritablement simple, nous devons souvent nous en remettre à de pures conjectures, et nous ne pou-

2. Nous employons ici - comme dans tous les exemples qui suivront - les crochets carrés pour désigner les propositions et les représentations; [X] désigne ainsi la représentation ou la proposition qui est le sens de l'expression «X». 
vons que dans très peu de cas présumer avec certitude qu'elle l'est ou qu'elle serait reconnue comme telle par Bolzano. Pour cette raison, il nous serait impossible d'appliquer la procédure de substitution à des cas concrets sous la clause (V1'), ce qui lui enlève pratiquement toute valeur. De plus, Bolzano luimême présente nombre d'exemples dans lesquels il varie ou substitue des représentations complexes. Nous rejetons donc (V1') comme inadéquate pour des raisons tant pratiques qu'historiques. Nous pouvons ainsi fixer notre seconde clause, qui consiste dans la négation de (V1') :

(V2) Dans $S\left(v_{1}, \ldots, v_{n} / w_{1}, \ldots, w_{n}\right), v_{1}, \ldots, v_{n}$ peuvent désigner des représentations qui ne sont pas simples.

\section{Troisième clause}

L'exigence selon laquelle les représentations qui sont substituées doivent non seulement être mutuellement exclusives mais aussi mutuellement disjointes, c'est-à-dire hétérogènes, nous conduit à des difficultés similaires à celles où nous conduit la clause (V1'), que nous venons de rejeter. On devrait définir ce concept de la manière suivante :

Les représentations ou propositions $l_{1}, \ldots, l_{n}$ sont mutuellement disjointes (ou hétérogènes) ssi aucune paire d'éléments distincts en $\left\{l_{1}, \ldots, l_{n}\right\}$ n'a d'élément commun.

Ou formellement :

Disjointes $\left(l_{1}, \ldots, l_{n}\right) \leftrightarrow$ $\forall l_{i} \forall l_{j}\left((1 \leq i \leq n \wedge 1 \leq j \leq n \wedge i \neq j) \rightarrow \neg \exists l\left(\operatorname{Teil}\left(l, l_{i}\right) \wedge \operatorname{Teil}\left(l, l_{j}\right)\right)\right)$

Une telle restriction rendrait, elle aussi, la procédure substitutionnelle bolzanienne inapplicable puisque, dans plusieurs cas, nous ne pourrions pas être certains que deux représentations ne contiennent pas, en fin de compte, une même représentation simple quelconque. Nous retenons donc :

(V3) Dans $S\left(v_{1}, \ldots, v_{n} / w_{1}, \ldots, w_{n}\right), v_{1}, \ldots, v_{n}$ peuvent désigner des représentations qui ne sont pas disjointes.

L'univocité de la procédure de substitution simultanée pourrait aussi, bien entendu, être garantie autrement que par la restriction (V1), à savoir en reconduisant cette restriction à la procédure de substitution uniforme simple d'après laquelle, dans une proposition $S$, une représentation unique $v$ est échangée pour une représentation $w: S(v / w)$. La procédure de substitution simultanée $S\left(v_{1}, \ldots\right.$, $v_{n}\left(w_{1}, \ldots, w_{n}\right)$ peut toujours être ramenée à une application $2 n$-fois successive de $S(v / w)$, avec quoi on garantit l'univocité sans clause restrictive pour $v_{1}, \ldots, v_{n}$. Cette voie est toutefois plutôt incommode dans la pratique et on l'évite en général pour cette raison. C'est aussi pourquoi j'en fait abstraction et emploie la procédure de substitution uniforme et simultanée comme concept primitif ${ }^{3}$.

3. Il est essentiel pour la définition du concept de validité universelle logique (et du concept de non validité universelle logique) d'employer la procédure de substitution simultanée 


\section{Quatrième clause}

Il est indispensable au concept d'analyticité logique que nous puissions faire la distinction entre les représentations ou propositions purement logiques et les représentations ou propositions qui ne sont pas purement logiques. "Cette distinction est à vrai dire vacillante, car le domaine des concepts qui appartiennent à la logique n'est pas si bien délimité qu'on ne puisse jamais soulever de dispute à ce propos ${ }^{4}$. On tranche généralement la question en énumérant les représentations logiques simples, les seules à partir desquelles toutes les représentations (purement) logiques complexes et toutes les propositions (purement) logiques sont structurées ${ }^{5}$.

On peut, sur la base des travaux de Bolzano, composer une liste imposante des concepts qu'il considérait logiques. Ils se répartissent (du point de vue contemporain) en deux groupes :

(L1) a) [avoir], [non-] ou [manque de], [et], [ou], ...

b) [quelque chose] ou [objet], [propriété], [collection],...

(L2) [proposition], [représentation], [vérité] ou [vrai], [fausseté] ou [faux], [objectualité] ou [objectuel], [non-objectualité] ou [non-objectuel], [déductibilité] ou [déductible].

(L1) regroupe les représentations logiques au sens étroit du terme. On peut y distinguer deux sous-classes :

(a) les exemples de (L1a) sont des «constantes logiques». En plus de la copule [avoir], on y retrouve aussi la représentation [non-], qui est le plus souvent identifiée à la "négation de propriété » [manque de] et à laquelle on peut reconduire, en vertu de l'identité [non-p] $=[[\mathrm{p}]$ a le manque de vérité], la négation propositionnelle. (Je doute toutefois que Bolzano trouve effectivement son compte dans une forme unique de négation; car d'après son analyse, la représentation [non-] est aussi contenue comme composante dans la représentation $[$ rien $]:[$ Nichts $]=$

$S\left(v_{1}, \ldots, v_{n} / w_{1}, \ldots, w_{n}\right)$, même si cette dernière peut être analysée en termes d'applications successives de la substitution simple $S(v / w)$. Dans tous les cas, la définition simplifiée qui surgit sans cesse dans la littérature secondaire est inadéquate : $S$ est logiquement universellement valide ssi pour au moins une représentation $v$ et pour au moins une représentation $w$ la représentationsujet de $S(v / w)$ est objectuelle et pour toute représentation $v$ et pour toute représentation $w$ la condition suivante s'applique : Si $v$ n'est pas (purement) logique et la représentation-sujet de $S(v / w)$ est objectuelle, alors $S(v / w)$ est vraie. D’après cette définition, la proposition manifestement synthétique [Si certains hommes sont des anges, alors certains êtres vivants sont des anges] serait logiquement analytique. Cet exemple trouve son origine dans R. Myhill et Benson Mates et est cité par Quine dans "Carnap and Logical Truth », Synthese, 12, 1960, p. 350-74, p. 352s.; voir la discussion dans Morscher, Edgar, «Bolzano's Method of Variation : Three Puzzles ", Grazer philosophische Studien, 53, 1997, p. 151-159.

4. Bolzano, Bernard, Wissenschaftslehre, Sulzbach, Seidel, 1837, $\$ 148$, vol. 2, p. 84.

5. Dans ce qui suit nous ferons l'économie du 'purement' pour désigner les propositions et les représentations (ou concepts) purement logiques; nous parlerons de propositions et de représentations (ou concepts) logiques. 
[non-quelque chose]); cette composante de [non-quelque chose] ne peut toutefois certainement pas être identifiée à [manque de].)

(b) Les exemples de (L1b) correspondent à ce qu'on a parfois appelé, en référence au niveau linguistique, les "mots universels" (au sens des «Allwörter» de Carnap); il s'agit là de déterminations catégoriales qui, dans une langue formelle, sont la plupart du temps désignées par l'usage de différents types de variables.

Mais Bolzano compte aussi au rang des représentations logiques les exemples qui se trouvent sous (L2) et que l'on pourrait désigner par « représentations métalogiques".

Dans la procédure de substitution bolzanienne il est généralement présupposé que les représentations $v_{1}, \ldots, v_{n}$ qui sont substituées dans $S\left(v_{1}, \ldots\right.$, $\left.v_{n} / w_{1}, \ldots, w_{n}\right)$ ne peuvent pas être d'espèce logique. Il n'y a pas à vrai dire de justification explicite pour cela chez Bolzano, mais il y a néanmoins une série d'indices. Aussi compréhensible que soit cette restriction pour les représentations logiques du groupe (L1), on voit toutefois mal pourquoi les représentations logiques du groupe (L2) devraient, elles aussi, être concernées. Mais, Bolzano voulait certainement exclure la copule d'une proposition c'est-à-dire la représentation logique [avoir] — soit variée; d'après Bolzano, seule les représentations-sujets et les représentations-prédicats d'une proposition, ou leurs composantes, peuvent être variées ${ }^{6}$.

Nous n'avons pas à régler ce problème ici, car il s'agit d'un problème qui n'est pas pertinent pour la question qui nous occupe. Seulement deux points sont essentiels dans le contexte présent, et à vrai dire, une chose avant tout : Même s'il n'est pas en principe permis de substituer les représentations logiques que contient une proposition, elles peuvent dans tous les cas servir pour remplacer des représentations qui sont considérées variables. Bolzano fait appel à plusieurs reprises à la représentation logique [quelque chose] pour les fins de la substitution ${ }^{7}$. Nous retenons par conséquent :

(V4) Dans $S\left(v_{1}, \ldots, v_{n} / w_{1}, \ldots, w_{n}\right), v_{1}, \ldots, v_{n}$ peuvent désigner des représentations logiques.

\section{Cinquième clause}

Nous pouvons de plus retenir que même si aucune des représentations $v_{1}, \ldots$, $v_{n}$ substituées dans $S\left(v_{1}, \ldots, v_{n} / w_{1}, \ldots, w_{n}\right)$ ne peut être une représentation logique (quelque en soit le sens), ces représentations peuvent néanmoins contenir une représentation logique comme composante :

(V5) Dans $S\left(v_{1}, \ldots, v_{n} / w_{1}, \ldots, w_{n}\right), v_{1}, \ldots, v_{n}$ peuvent désigner des représentations qui contiennent comme composantes des représentations logiques.

6. Größenlehre, dans Bernard Bolzano-Gesamtausgabe, dir. E. Winter, J. Berg, F. Kambartel, J. Louzil, B. van Rootselaar, Stuttgart-Bad Cannstatt, 2 A 7, p. 62.

7. Par exemple, Bolzano, 1837, \$197, vol. 2, p. 333. 
La clause (V5) est étroitement liée à (V2) : Nous aurions de très bonnes raisons de croire que des représentations logiques sont toujours nécessaires à la composition des représentations complexes en tant que «liant» ou "mastic »; sans un tel «mastic» logique, les parties d'une représentation complexe — pour parler métaphoriquement - ne tiennent pas ensemble. Les représentations complexes contiennent donc — si ce n'est pas sans exception c'est pour le moins en général - des représentations logiques comme composantes. Sans la clause (V5), nous encourrions le risque de devoir aussi abandonner la clause (V2).

\section{Sixième clause}

La procédure de substitution $S\left(v_{1}, \ldots, v_{n} / w_{1}, \ldots, w_{n}\right)$ est aussi définie pour le cas où les représentations $v_{1}, \ldots, v_{n}$ ne sont nullement contenues dans $S$ :

(V6) Dans $S\left(v_{1}, \ldots, v_{n} / w_{1}, \ldots, w_{n}\right), v_{1}, \ldots, v_{n}$ peuvent désigner des représentations qui ne sont pas contenues dans $S$.

Si aucune des représentations $v_{1}, \ldots, v_{n}$ n'est contenue dans $S$, alors $S\left(v_{1}, \ldots, v_{n} / w_{1}, \ldots, w_{n}\right)$ est, dans tous les cas — c'est-à-dire indépendamment du choix de $w_{1}, \ldots, w_{n}$, identique à $S$ :

$(\mathrm{V} 6 *)$ Si aucune représentation $v_{1}, \ldots, v_{n}$ n'est contenue dans $S$, alors pour toute représentation $w_{1}, \ldots, w_{n}: S\left(v_{1}, \ldots, v_{n} / w_{1}, \ldots, w_{n}\right)=S$.

\section{L'interprétation courante}

On définit habituellement le concept bolzanien de l'analyticité logique de la manière suivante :

(A1) Une proposition $S$ est logiquement analytique ssi $S$ est logiquement universellement valide ou logiquement universellement non valide.

(A2) Une proposition $S$ est logiquement universellement valide ssi $S$ est universellement valide à l'égard de toutes les représentations $v_{1}, \ldots, v_{n}$ qui, dans $S$, sont non logiques.

(A3) Une proposition $S$ est logiquement universellement non valide ssi $S$ est universellement non valide à l'égard de toutes les représentations $v_{1}, \ldots$, $v_{n}$ qui, dans $S$, sont non logiques.

L'expression " universellement valide à l'égard des représentations $v_{1}, \ldots$, $v_{n}$ » utilisée dans le definiens de (A2) peut être définie de la manière suivante :

(A4) Une proposition $S$ est universellement valide à l'égard des représentations mutuellement exclusives $v_{1}, \ldots, v_{n}$ ssi

(i) il y a au moins une proposition qui résulte de la substitution uniforme et simultanée de $v_{1}, \ldots, v_{n}$ dans $S$ pour des représentations de même catégorie dont la représentation-sujet est objectuelle (gegenständlich); et

(ii) toutes les propositions qui résultent de la substitution uniforme et simultanée de $v_{1}, \ldots, v_{n}$ dans $S$ pour des représentations de même catégorie et dont la représentation-sujet est objectuelle sont vraies; 
c'est-à-dire ssi la condition suivante s'applique :

(ii) pour au moins une suite de représentations $w_{1}, \ldots, w_{n}$ de même catégorie que $v_{1}, \ldots, v_{n}$, la représentation-sujet de $S\left(v_{1}, \ldots, v_{n} / w_{1}, \ldots, w_{n}\right)$ est objectuelle, et

(ii) pour toute suite de représentations $w_{1}, \ldots, w_{n}$ de même catégorie que $v_{1}, \ldots, v_{n}$, si la représentation-sujet de $S\left(v_{1}, \ldots, v_{n} / w_{1}, \ldots, w_{n}\right)$ est objectuelle, alors $S\left(v_{1}, \ldots, v_{n} / w_{1}, \ldots, w_{n}\right)$ est vraie.

On définit " universellement non valide à l'égard de $v_{1}, \ldots, v_{n}$ " de manière analogue :

(A5) Une proposition est universellement non valide à l'égard des représentations mutuellement exclusives $v_{1}, \ldots, v_{n}$ ssi pour toute représentation $w_{1}, \ldots, w_{n}$ de même catégorie que $v_{1}, \ldots, v_{n}, S\left(v_{1}, \ldots, v_{n} / w_{1}, \ldots, w_{n}\right)$ est fausse.

\section{Un problème technique avec $\left(A_{2}\right)$ et $\left(A_{3}\right)$}

Revenons d'abord, pour les fins de l'explication, à notre exemple initial : $S_{1}=$ [Tout homme judicieux est judicieux]. Je renonce à transposer cet exemple dans la forme canonique bolzanienne [A a b] — la traduction est sans cela évidente. $S_{1}$ est manifestement logiquement universellement valide. Les composantes non logiques de $S_{1}$ sont les représentations suivantes : [homme], [judicieux], [homme judicieux]. L'ensemble $M_{1}=$ [[homme], [judicieux], [homme judicieux]\} de toutes les composantes non logiques de $S$ n'est toutefois pas mutuellement exclusif, et c'est pourquoi la procédure de substitution simultanée n'est pas définie pour tous les éléments de $M_{1}$ en même temps. Mais, manifestement, il suffit dans le cas présent que $S_{1}$ soit universellement valide à l'égard de l'ensemble mutuellement exclusif $M_{1}^{\prime}{ }_{1}=\{$ homme], [judicieux] $; M_{1}^{\prime}$ est dans un certain sens un ensemble de toutes les composantes mutuellement exclusives non logiques de $S_{1}$, et ce dans la mesure où l'adjonction d'un élément additionnel de $M_{1}$ à $M_{1}^{\prime}$ mène à ceci que les éléments de ce nouvel ensemble (en l'occurrence $M_{1}$ lui-même) ne sont plus mutuellement exclusifs. Il ressort de cette suggestion que (A2) devrait être modifié de la manière suivante :

(A2') Une proposition $S$ est logiquement universellement valide ssi $S$ est universellement valide à l'égard d'un ensemble maximalement exclusif de représentations non logiques.

Cette modification serait néanmoins vaine, et ce pour la raison suivante : $M{ }_{1}=\{[$ homme judicieux $]\}$ est aussi un ensemble maximalement exclusif de composantes non logiques de $S_{1}$ dans la mesure où l'adjonction d'un élément additionnel de $M_{1}$ à $M^{\prime}{ }_{1}$ donne un ensemble dont les éléments ne sont pas mutuellement exclusifs. Afin de sauvegarder l'interprétation standard, une autre modification de (A2) est indispensable. À cet égard, la difficulté consiste en ceci qu'on ne peut pour cela se rapporter à l'expression linguistique de la proposition sans trahir le caractère "purement objectif » 
de la logique bolzanienne. Seule est admise le recours à la décomposition des propositions. De la sorte, on peut déterminer de manière "purement objective » ce qu'implique l'idée qu'une représentation $v$ "apparaît à différentes places » dans une proposition $S$; cela ne veut rien dire de plus que ceci que plusieurs membres de la décomposition de $S$ sont identiques à $v$. On peut difficilement parvenir de la sorte à une modification appropriée de (A2) qui soit à la fois simple et naturelle. Cela apparaît, finalement, à la lumière de l'exemple quelque peu compliqué de la proposition logiquement universellement valide $\mathrm{S}_{2}$ :

S2 = [Toute personne mâle adulte non mariée est une personne mâle non mariée]

On peut d'abord décomposer $S_{2}$, même sans la transposer dans la forme bolzanienne [A a b], en une représentation-sujet [personne mâle adulte non mariée] et une quasi-représentation-prédicat [personne mâle non mariée]; on peut ensuite décomposer ces deux représentations dans leurs composantes immédiates, et procéder de la même manière avec ces dernières, jusqu'à ce que qu'on parvienne aux représentations [personne], [mâle], [adulte] et [mariée], lesquelles nous ne voulons pas décomposer plus avant pour des raisons de simplicité. (Une décomposition exhaustive devrait progresser jusqu'aux représentations simples). Nous réunissons les représentations non logiques qui résultent de la décomposition de $S_{2}$ dans l'ensemble $M_{2}$ :

$M_{2}=\{[$ personne mâle adulte non mariée], [personne mâle non mariée], [personne mâle adulte], [personne mâle], [non-marié] [personne], [mâle], [adulte],[mariée]\}

D'après (A2), « $S_{2}$ est logiquement universellement valide» veut dire que $S_{2}$ est universellement valide à l'égard d'une sélection déterminée d'éléments de $M_{2}$ qui sont mutuellement exclusifs et qui dans un certain sens «couvrent» bel et bien tous les éléments de $M_{2}$. Les sous-ensembles suivants sont des ensembles de composantes non logiques de $S_{2}$ qui sont maximalement exclusives et qui peuvent, sans problème pour la définition (A2), être mis à contribution : dans chaque cas la définition (A2) livre le résultat souhaité, à savoir que $S_{2}$ est logiquement universellement valide :

$M_{2}^{1}=\{[$ personne], [mâle], [adulte], [mariée] $\}$

$M_{2}^{2}=\{[$ personne], [mâle], [adulte], [non mariée] $\}$

$M_{2}^{3}=\{[$ personne mâle], [adulte], [mariée] $\}$

$M_{2}^{4}=\{[$ personne mâle], [adulte], [non mariée $]\}$

Les deux sous-ensembles de $M_{2}$ qui suivent sont aussi des ensembles maximalement exclusifs de composantes non logiques de $\mathrm{S}_{2}$, mais ils sont pour les fins de (A2) inutilisables :

$M_{2}^{5}=\{[$ personne mâle adulte non mariée], [personne mâle non mariée] $\}$

$M_{2}^{6}=\{[$ personne mâle adulte $],[$ non mariée $]\}$ 
La raison pour laquelle $M_{2}^{5}$ et $M_{2}^{6}$ ne conviennent pas au but visé est intuitivement claire : $S_{2}$ n'est nullement universellement valide à l'égard des éléments de $M_{2}^{5}$ et $M_{2}^{6}$; et lorsqu'on applique la procédure substitutionnelle à $S_{2}$ à l'égard des éléments de $M_{2}^{6}$, une des parties non logiques de $S_{2}$ reste même inchangée (à savoir [personne mâle]). Le problème technique en ce qui concerne la définition bolzanienne de la validité universelle logique consiste à fournir un critère praticable pour cette distinction intuitivement claire sans pour cela faire référence à l'expression linguistique des propositions (et en particulier sans faire référence à la simplicité de certaines représentations). Le problème que nous venons d'indiquer en nous appuyant sur (A2) se pose bien entendu aussi de la même manière pour (A3).

Cependant il ne vaut pas la peine de chercher à résoudre ce problème, car les définitions (A1)-(A3) souffrent, quant à leur contenu, d'une lacune dont je discute en détail dans la prochaine section.

\section{L'impropriété des définitions $\left(A_{1}\right)-\left(A_{3}\right)$}

L'impropriété de (A1)-(A3) se montre au jour dès qu'on tente de les appliquer à des propositions logiques. Une proposition est logique si et seulement si elle est composée exclusivement de représentations logiques; si, donc, tant sa représentation-sujet que sa représentation-prédicat sont logiques. Nous avons distingué deux groupes de représentations logiques. D’après Bolzano, le concept [avoir] constitue la copule de toute proposition, et donc de toute proposition logique. Le concept [avoir] appartient aux représentations logiques au sens étroit, à savoir au groupe (L1) des représentations logiques. Lorsque tant la représentation-sujet que la représentation-prédicat d'une proposition appartient au groupe (L1), nous comptons la proposition en question au sein du groupe (L1*) des propositions logiques. Nous distinguons ce dernier du groupe $(\mathrm{L} 2 *)$ des propositions logiques, dont la représentation-sujet et/ou la représentation-prédicat appartiennent au groupe (L2). On peut aussi appeler les propositions logiques du groupe (L2*) «propositions métalogiques» et on peut souligner parmi elles les propositions purement métalogiques, c'est-à-dire celles dont tant la représentation-sujet que la représentation-prédicat appartiennent au groupe (L2). Voici quelques exemples de telles propositions métalogiques :

[Toute représentation est non objectuelle]

[Toute proposition est vraie]

[Toute proposition dont la représentation-sujet est non objectuelle est fausse]

etc.

Une proposition dont la représentation-sujet est [proposition], [représentation], [proposition vraie] ou [représentation non objectuelle] et dont la représentation-prédicat appartient, elle aussi, aux représentations métalogiques du groupe (L2) telles que [vrai], [faux], [objectuel], [non objectuelle] est donc aussi dans tous les cas métalogiques. 
Il n'est pas aussi simple de trouver des exemples nets de propositions logiques de l'espèce (L1*). Cela découle du fait que Bolzano interprète certains types de propositions - telles que les propositions existentielles, les propositions conjonctives et les propositions disjonctives - comme étant des propositions métalogiques. Ainsi, par exemple, la proposition [Il y a une licorne] est d'après Bolzano identique avec la proposition métalogique [La représentation [licorne] est objectuelle]. C'est pourquoi la proposition [Il y a quelque chose] même si elle semble prima facie appartenir au groupe (L1*) est toutefois, conformément à l'analyse bolzanienne, identique à [La représentation [quelque chose] est objectuelle], et appartient donc, par conséquent, bel et bien au groupe (L2*). Cette classification dépend toutefois de l'analyse particulière que fait Bolzano de telles propositions. Je rassemble les propositions qui devraient normalement appartenir à (L1*) mais qui, en vertu de l'analyse particulière de Bolzano, sont classées au sein du groupe (L2*) dans un groupe distinctif : (L1/2*).

On retrouve indubitablement dans les trois groupes de propositions logiques des exemples de propositions logiquement universellement valides, des exemples de propositions logiquement universellement non valides de même que des exemples de propositions qui ne sont ni l'une, ni l'autre.

Des exemples de propositions logiques qui sont logiquement universellement valides sont :

(L1*) [Tout objet est un objet] [Toute propriété est une propriété]

$(\mathrm{L} 1 / 2 *)$ [Il y a quelque chose ou il n'est pas le cas qu'il y a quelque chose]

(L2*) [Toute représentation qui est objectuelle est objectuelle]

[Toute proposition vraie est vraie]

[Il y a une représentation non objectuelle ou il n'est pas le cas qu'il y a une représentation non objectuelle]

[Toute proposition est vraie ou il n'est pas le cas que toute proposition est vraie].

On peut former des exemples de propositions logiques qui sont logiquement universellement non valides en niant les exemples de propositions logiquement universellement valides.

Des exemples de propositions logiques qui ne sont ni logiquement universellement valides ni logiquement universellement non valides sont :

(L1*) [Toute propriété est un objet]

[Tout objet a au moins une propriété]

$(\mathrm{L} 1 / 2 *)[\text { Il y a quelque chose }]^{8}$

[Il y a un nombre infini d'objets]

8. La proposition prise en exemple : [Il y a quelque chose] appelle quelques réflexions additionnelles.

(i) L'analyse bolzanienne admet [Il y a quelque chose] = [[quelque chose] a l'objectualité]. La représentation-sujet de cette proposition n'est donc nullement [quelque chose] 
(L2*) [Il y a une représentation non objectuelle]

[Il y a une proposition vraie]

[Toute représentation est objectuelle]

[Toute proposition est vraie].

Les définitions (A1)-(A3) achoppent lorsqu'on les applique à des propositions logiques de cette espèce. Ces propositions ne contiennent en vérité que des composantes logiques et aucune représentation non logique. D'après (A2) et (A3), il en va de savoir si ces propositions sont universellement valides ou universellement non valides à l'égard de l'ensemble des représentations non logiques $v_{1}, \ldots, v_{n}$ qui s'y trouvent. Quelles que soient les représentations non logiques $v_{1}, \ldots, v_{n}$ que nous choisissions, elles ne peuvent nullement se trouver dans une proposition logique. Mais si aucune représentation $v_{1}, \ldots, v_{n}$ n'est contenue dans une proposition $S$, il n'y a alors à l'égard de $v_{1}, \ldots, v_{n}$ qu'une seule variante de $S$, à savoir $S$ elle-même : $S\left(v_{1}, \ldots, v_{n} / w_{1}, \ldots, w_{n}\right)$ est donc, en l'occurrence, en vertu de $\left(\mathrm{V}^{*}\right)$, identique à $S$ pour toute suite de représentations $w_{1}, \ldots, w_{n}$. À supposer qu'elles soient vraies, les propositions logiques sont donc trivialement universellement valides à l'égard des représentations non logiques (qui ne sont même pas contenues en elles). Si elles sont fausses, elles sont trivialement universellement non valides. Par conséquent, elles sont toutes — conformément à (A2) et (A3) - trivialement logiquement universellement valides ou non valides et par suite - conformément à (A1) - logiquement analytiques.

Il suit donc des définitions (A1)-(A3), si tant est que nous autorisions leur application à des propositions logiques, que toute proposition logique est logiquement analytique, c'est-à-dire qu'elle est soit logiquement universellement valide, soit logiquement universellement non valide.

Afin de neutraliser cette conséquence, on devrait concevoir les définitions (A1)-(A3) comme des définitions conditionnelles et mettre en tête de chacune d'entre elles la condition :

Si $S$ n'est pas une proposition logique, alors la définition suivante s'applique :...

mais [la représentation [quelque chose ]] ou — exprimé de manière plus concise [[quelque chose]]. Même si [quelque chose] est une représentation logique, cela ne veut pas automatiquement dire que [[quelque chose]] l'est aussi. On doit pour cela supposer un principe supplémentaire, qui apparaît tout à fait plausible et qui peut être formulé de la manière suivante : si la représentation [...] est logique, alors la représentation [[...]] l'est aussi. La proposition [[quelque chose] a l'objectualité] appartient dans tous les cas au deuxième groupe (c'est-à-dire aux propositions métalogiques) et non pas au premier groupe des propositions logiques.

(ii) D'après Bolzano, la proposition [Il y a quelque chose] est une vérité primitive (Bolzano, 1837, \$214, vol.2, p.375) et, par conséquent, elle est vraie. Parce qu'elle ne contient - attendu que ce que nous avons dit en (i) est vrai - aucune composante non logique, on n'obtient jamais, par la variation de ses composantes non logiques, que cette même proposition (vraie), et ce de manière triviale. Si on suit l'interprétation courante, cette proposition serait aussi universellement valide, ce qui n'est manifestement pas la position de Bolzano. 
Le cas échant, les définitions (A1)-(A3) ne seraient nullement applicables aux propositions logiques, et nous ne pourrions plus distinguer, parmi les propositions logiques, les propositions logiquement universellement valides, les propositions logiquement universellement non valides et celles qui ne sont ni l'une ni l'autre. Mais il y a - comme nous l'avons indiqué plus haut - parmi les propositions logiques des exemples distincts des trois catégories.

Les définitions (A1)-(A3) se révèlent donc inadéquates. Et il ne sert à rien de nous écarter de Bolzano et de ne pas compter les représentations et propositions métalogiques au rang des représentations et propositions logiques, car nous pouvons fournir pour les trois catégories ( "logiquement universellement valide ", "logiquement universellement non valide» et "ni l'un ni l'autre») des exemples univoques de propositions logiques qui n'appartiennent pas au groupe métalogique (L2*). C'est d'ailleurs pour tirer ceci au clair (et seulement pour cette raison) que j'ai distingué les trois groupes de propositions logiques.

Il est ainsi établi que les définitions (A1)-(A3) et, par conséquent, une interprétation du concept bolzanien d'analyticité logique amplement répandue dans la littérature sont inadéquates. Aussi chercherons-nous maintenant une reconstruction adéquate de la définition bolzanienne selon laquelle une proposition logique est une instance substitutionnelle d'une forme propositionnelle générée à partir d'une proposition non logiquement analytique. Il est à cette fin indispensable que, dans la procédure de substitution $S\left(v_{1}, \ldots\right.$, $v_{n}\left(w_{1}, \ldots, w_{n}\right)$, des représentations logiques assument une fonction substitutive et qu'elles puissent être substituées aux représentations non logiques $v_{1}, \ldots, v_{n}$; ce qui, conformément à (V4), est admissible.

\section{Suggestions pour une reconstruction du concept bolzanien d'analyticité logique.}

6.1. Exposition informelle

\subsubsection{Variables}

Les quatre espèces de variables suivantes seront utilisées dans notre reconstruction :

(1) Variables pour des objets quelconques : $x, y, z, \ldots$

(2) Variables pour les représentations : $v, v^{\prime}, v_{1}, v_{2}, \ldots, w, w^{\prime}, w_{1}, w_{2}, \ldots$

(3) Variables pour les propositions : $S, S^{\prime}, S_{1}, S_{2}, \ldots$

(4) Variables pour les objets logiques quelconques (c'est-à-dire pour les propositions ou les représentations) $: l, l, l_{1}, l_{2}, \ldots, m, m^{\prime}, m_{1}, m_{2}, \ldots$ 


\subsubsection{Concepts primitifs non définis}

Le foncteur suivant et les sept prédicats introduits à sa suite sont posés comme concepts primitifs non définis :

(1) la proposition qui est générée à partir de la proposition $S$ par la substitution uniforme et simultanée des représentations mutuellement exclusives $v_{1}, \ldots, v_{n}$ par les représentations $w_{1}, \ldots, w_{n}$

(2) la représentation ou la proposition $l_{1}$ est une composante immédiate de la représentation ou de la proposition $l_{2}$

(3) les représentations $v_{1}$ et $v_{2}$ appartiennent à la même catégorie ou $v_{1}$ et $v_{2}$ sont équicatégoriales

(4) la représentation $v$ est la représentation-sujet de la proposition $S$

(5) la représentation $v$ est objectuelle

(6) la proposition $S$ est vraie

(7) la proposition $S$ est fausse

(8) $v$ est une représentation logique.

Le fait que ces expressions assument la fonction de concepts primitifs et qu'ils restent pour cette raison non-définis dans le cadre de la présente investigation ne signifie pas qu'ils soient en principe indéfinissables ou qu'ils n'aient pas été définis par Bolzano; au contraire, plusieurs de ces termes, comme par exemple "vrai » et "faux », sont définissables et Bolzano les a effectivement définis.

\subsubsection{Définitions}

(D1) Une séquence décompositionnelle — ou en bref : une décomposition - d'une représentation ou d'une proposition $l$ est une suite de représentations et/ou de propositions dont le premier membre est $l$ luimême et pour laquelle la condition suivante s'applique : chaque membre de la suite - à l'exception du premier membre, c'est-à-dire $l$ - est partie immédiate d'un membre qui, dans cette suite, le précède, et toutes les parties immédiates d'un membre le suivent.

Remarques :

(i) Une séquence décompositionnelle ou décomposition est l'inverse d'une séquence de formation.

(ii) être la composante immédiate d'une proposition ou d'une représentation est l'inverse de : être formé à partir d'une représentation ou d'une proposition d'une manière déterminée (c'est-à-dire d'après une certaine règle de formation)

(iii) D'après Bolzano une séquence décompositionnelle s'achève avec des représentations simples. 
(iv) Une représentation ou proposition peut avoir différentes décompositions. Lorsqu'on fait abstraction des différences inessentielles (comme l'ordre des membres) ou lorsqu'on opère certaines restrictions conventionnelles ou standardisations, on peut attribuer de manière univoque à toute représentation ou proposition une séquence décompositionnelle et ensuite parler de la décomposition d'une représentation ou d'une proposition.

(D2) Une partie d'une représentation ou d'une proposition $l$ est un membre de la décomposition de $l$.

Remarque : D'après Bolzano, toutes les décompositions d'une représentation ou d'une proposition ont les mêmes membres, elles ne se distinguent les unes des autres qu'en vertu de l'ordre de leurs membres; cf. Remarque (iv) de (D1).

(D3) Les représentations ou propositions $l_{1}, \ldots, l_{n}$ s'excluent l'une l'autre mutuellement (ou elles sont mutuellement exclusives) ssi aucun élément de $\left\{l_{1}, \ldots, l_{n}\right\}$ n'est membre de l'un des éléments de $\left\{l_{1}, \ldots, l_{n}\right\}$.

(D4) La forme d'une proposition $S$ à l'égard des représentations mutuellement exclusives $v_{1}, \ldots, v_{n}$, dont au moins une est contenue comme partie de $S$ est l'ensemble des propositions qui sont générées à partir de la substitution uniforme et simultanée de $v_{1}, \ldots, v_{n}$ par des représentations $w_{1}, \ldots, w_{n}$ qui leur sont équicatégoriales.

Remarque : La première condition du definiens de (D4) exclut qu'une forme d'une proposition $S$ ne consiste qu'en elle-même ou qu'elle soit identique avec l'ensemble unaire $\{S\}$; ce cas "dégénéré » d'une «forme» dans lequel une procédure substitutionnelle «fonctionne à vide» est écarté par (D4).

(D5) Une forme d'une proposition $S$ est la forme de $S$ à l'égard d'une suite de représentations mutuellement exclusives $v_{1}, \ldots, v_{n}$.

(D6) Une forme propositionnelle — ou, en bref, une forme - est une forme d'une proposition.

(D7) Une instance substitutionnelle — ou, en bref, une substitution — d'une forme propositionnelle est un élément d'une forme propositionnelle.

(D8) Une forme propositionnelle est universellement valide ssi au moins une de ses instances substitutionnelles a une représentation-sujet objectuelle et toutes ses instances substitutionnelles dont la représentationsujet est objectuelle sont vraies.

(D9) Une forme propositionnelle est universellement non valide lorsque toutes ses instances substitutionnelles sont fausses.

(D10) Une forme propositionnelle logique est une forme propositionnelle dans laquelle toutes les représentations contenues comme parties dans tous ses éléments sont des représentations logiques. 
(D11) Une proposition $S$ est universellement valide à l'égard des représentations mutuellement exclusives $v_{1}, \ldots, v_{n}$ ssi la forme propositionnelle qui est la forme de $S$ à l'égard de $v_{1}, \ldots, v_{n}$ est universellement valide. Remarque : d'après cette définition — de même que d'après l'interprétation courante - , il y a des propositions universellement valides qui ont une représentation-sujet non-objectuelle et qui sont donc fausses. Si nous voulons parvenir à ce que toute proposition universellement valide à l'égard de $v_{1}, \ldots, v_{n}$ soit aussi toujours vraie, nous devons compléter le définiens par la condition suivante : la représentation-sujet de $S$ est objectuelle ou $S$ est vrai.

(D12) Une proposition $S$ est universellement non valide à l'égard des représentations mutuellement exclusives $v_{1}, \ldots, v_{n}$ ssi la forme de $S$ est universellement non valide à l'égard de $v_{1}, \ldots, v_{n}$.

(D13) Une proposition $S$ est analytique à l'égard des représentations mutuellement exclusives $v_{1}, \ldots, v_{n}$ ssi $S$ est universellement valide ou universellement non valide à l'égard de $v_{1}, \ldots, v_{n}$.

(D14) Une proposition $S$ est synthétique à l'égard des représentations mutuellement exclusives $v_{1}, \ldots, v_{n}$ ssi $S$ n'est pas analytique, donc ni universellement valide, ni universellement non valide à l'égard de $v_{1}, \ldots, v_{n}$.

(D15) Une proposition $S$ est universellement valide ssi $S$ (ou la forme de $S$ ) est universellement valide à l'égard d'au moins une suite de représentations mutuellement exclusives $v_{1}, \ldots, v_{n}$.

D’après notre définition, une proposition universellement valide peut avoir une représentation-sujet non objectuelle et donc être fausse. Pour éviter cela, nous devons ici, ou dans la définition (D11,) compléter le definiens par une condition appropriée; voir la remarque de (D11).

(D16) Une proposition $S$ est universellement non valide ssi $S$ (ou la forme de $S$ ) est universellement non valide à l'égard d'une suite de représentations mutuellement exclusive $v_{1}, \ldots, v_{n}$.

(D17) Une proposition $S$ est analytique ssi $S$ est universellement valide ou universellement non valide, c'est-à-dire si $S$ est analytique et donc universellement valide ou non valide à l'égard d'au moins une suite de représentations mutuellement exclusives $v_{1}, \ldots, v_{n}$.

(D18) Une proposition $S$ est synthétique ssi $S$ n'est pas analytique et par conséquent ni universellement valide, ni universellement non valide

(D19) Une proposition $S$ est logiquement universellement valide ssi $S$ est une instance substitutionnelle d'une forme propositionnelle (purement) logique qui est universellement valide.

Remarque : Une proposition logiquement universellement valide peut aussi, en vertu de notre définition, avoir une représentation-sujet non 
objectuelle et par conséquent être fausse. Afin d'exclure cette possibilité, nous devons compléter le definiens de (D19) par la condition suivante : $S$ est vrai ou la représentation-sujet de $S$ est objectuelle. Bien entendu, un complément approprié à la définition (D11) suffit aussi — voir la remarque à cet endroit. Comme nous l'avons déjà mentionné, notre définition ne diffère pas sur ce point de l'interprétation courante.

(D20) Une proposition $S$ est logiquement universellement non valide ssi $S$ est une instance substitutionnelle d'une forme propositionnelle (purement) logique qui est universellement non valide.

(D21) Une proposition $S$ est logiquement analytique ssi $S$ est logiquement universellement valide ou logiquement universellement non valide, c'est-à-dire si $S$ est une instance substitutionnelle d'une forme logique qui est universellement valide ou non valide.

(D22) Une proposition $S$ est logiquement synthétique ssi $S$ n'est pas logiquement analytique et par conséquent ni logiquement universellement non valide, ni logiquement universellement non valide.

\subsection{Exposition formelle}

\subsubsection{Variables}

Les mêmes types de variables qu'au point 6.1.1. seront utilisées :

(1) Variables pour des objets quelconques : $x, y, z, \ldots$

(2) Variables pour les représentations : $v, v^{\prime}, v_{1}, v_{2}, \ldots, w, w^{\prime}, w_{1}, w_{2}, \ldots$

(3) Variables pour les propositions : $S, S^{\prime}, S_{1}, S_{2}, \ldots$

(4) Variables pour les objets logiques quelconques (c'est-à-dire pour les propositions ou les représentations) $: l, l, l_{1}, l_{2}, \ldots, m, m^{\prime}, m_{1}, m_{2}, \ldots$

\subsubsection{Concepts primitifs non définis}

Nous utilisons les symboles linguistiques suivants comme abréviations pour les concepts non définis introduit à 6.1.2. (dans le même ordre qu'à 6.1.2.) :
(1) $S\left(v_{1}, \ldots, v_{n} / w_{1}, \ldots, w_{n}\right)$
(2) $\operatorname{ComposIm}\left(l_{1}, l_{2}\right)$
(3) Équicatégoriales $\left(v_{1}, v_{2}\right)$
(4) $\operatorname{Sujet}(v, S)$
(5) Objectuelle $(v)$
(6) $\operatorname{Vraie}(S)$
(7) Fausse $(S)$
(8) $\operatorname{Logique}(v)$ 


\subsubsection{Définitions}

(D1) Décomposition $(x, l): \leftrightarrow \exists l_{1} \ldots \exists l_{n}\left[x=\left\langle l_{1}, \ldots, l_{n}\right\rangle \wedge l_{1}=l \wedge\right.$ $\forall i\left(1<i \leq n \rightarrow \exists j\left(j<i \wedge \operatorname{ComposIm}\left(l_{i}, l_{j}\right)\right)\right) \wedge \forall i \forall m((1 \leq i \leq n \wedge$ $\left.\left.\left.\operatorname{ComposIm}\left(m, l_{i}\right)\right) \rightarrow \exists j\left(i<j \leq n \wedge l_{j}=m\right)\right)\right]$

(D2) Partie $\left(l_{1}, l_{2}\right): \leftrightarrow \exists x \exists m_{1} \ldots \exists m_{n}\left[x=\left\langle m_{1}, \ldots, m_{n}\right\rangle \wedge\right.$ Décomposition $(x$, $\left.\left.l_{2}\right) \wedge \exists i\left(1 \leq i \leq n \wedge m_{i}=l_{1}\right)\right]$

(D3) Exclusives $\left(l_{1}, \ldots, l_{n}\right): \leftrightarrow \forall i \forall j[(1 \leq i \leq n \wedge 1 \leq j \leq n \wedge i \neq j) \rightarrow$ $\left.\neg \operatorname{Partie}\left(l_{i}, l_{j}\right)\right]$

(D4) Exclusives $\left(v_{1}, \ldots, v_{n}\right) \rightarrow\left[\operatorname{Forme}\left(x, S, v_{1}, \ldots, v_{n}\right): \leftrightarrow \exists i(1 \leq i \leq n \wedge\right.$ $\left.\operatorname{Partie}\left(v_{i}, S\right)\right) \wedge$ $\forall S^{\prime}\left[S^{\prime} \in x \leftrightarrow \exists w_{1} \ldots \exists w_{n}\left(\forall i\left(1 \leq i \leq n \wedge\right.\right.\right.$ Équicatégoriales $\left.\left(v_{i}, w_{i}\right)\right) \rightarrow$ $\left.\left.\left.S^{\prime}=S\left(v_{1}, \ldots, v_{n} / w_{1}, \ldots, w_{n}\right)\right)\right]\right]$

(D5) $\operatorname{Forme}(x, S): \leftrightarrow \exists v_{1} \ldots \exists v_{n}\left(\operatorname{Forme}\left(x, S, v_{1}, \ldots, v_{n}\right)\right)$

(D6) Forme $(x): \leftrightarrow \exists S(\operatorname{Forme}(x, S))$

(D7) $\operatorname{InstSub}(S, x): \leftrightarrow \operatorname{Forme}(x) \wedge S \in x$

(D8) VForme $(x): \leftrightarrow \operatorname{Forme}(x) \wedge \exists S(S \in x \wedge \operatorname{Objectuelle}(v) \wedge(\operatorname{Sujet}(v$, $S)) \wedge$ $\forall S[(S \in x \wedge \operatorname{Objectuelle}(v) \wedge(\operatorname{Sujet}(v, S))) \rightarrow \operatorname{Vraie}(S)]$

(D9) nonVForme $(x): \leftrightarrow$ Forme $(x) \wedge \forall S(S \in x \rightarrow \operatorname{Fausse}(S))$

$(\mathrm{D} 10) \operatorname{LForme}(x): \leftrightarrow \operatorname{Forme}(x) \wedge \forall v[\forall S(S \in x \rightarrow \operatorname{Partie}(v, S)) \rightarrow$ Logique $(v)]$

(D11) Exclusives $\left(v_{1}, \ldots, v_{n}\right) \rightarrow\left[\mathrm{V}\left(S, v_{1}, \ldots, v_{n}\right): \leftrightarrow \exists x\left(\operatorname{Forme}\left(x, S, v_{1}, \ldots\right.\right.\right.$, $\left.\left.\left.v_{n}\right) \wedge \operatorname{VForme}(x)\right)\right]$

(D12) Exclusives $\left(v_{1}, \ldots, v_{n}\right) \rightarrow\left[\operatorname{nonV}\left(S, v_{1}, \ldots, v_{n}\right): \leftrightarrow \exists x(\right.$ Forme $(x, S$, $\left.v_{1}, \ldots, v_{n}\right) \wedge$ nonVForme $\left.\left.(x)\right)\right]$

(D13) Exclusives $\left(v_{1}, \ldots, v_{n}\right) \rightarrow\left[\mathrm{A}\left(S, v_{1}, \ldots, v_{n}\right): \leftrightarrow \mathrm{V}\left(S, v_{1}, \ldots, v_{n}\right) \vee \operatorname{nonV}(S\right.$, $\left.\left.v_{1}, \ldots, v_{n}\right)\right]$

(D14) Exclusives $\left(v_{1}, \ldots, v_{n}\right) \rightarrow\left[S\left(S, v_{1}, \ldots, v_{n}\right): \leftrightarrow \neg \mathrm{A}\left(S, v_{1}, \ldots, v_{n}\right)\right]$

$(\mathrm{D} 15) \mathrm{V}(S): \leftrightarrow \exists v_{1} \ldots \exists v_{n}\left(\mathrm{~V}\left(S, v_{1}, \ldots, v_{n}\right)\right)$

$(\mathrm{D} 16) \operatorname{nonV}(S): \leftrightarrow \exists v_{1} \ldots \exists v_{n}\left(\operatorname{nonV}\left(S, v_{1}, \ldots, v_{n}\right)\right)$

(D17) $\mathrm{A}(S): \leftrightarrow \mathrm{V}(S) \vee \operatorname{nonV}(S)$

(D18) $S(S): \leftrightarrow \neg \mathrm{A}(S)$

$(\mathrm{D} 19) \operatorname{LV}(S): \leftrightarrow \exists x(\operatorname{LForme}(x) \wedge \operatorname{VForme}(x) \wedge \operatorname{InstSub}(S, x))$

$(\mathrm{D} 20) \operatorname{LnonV}(S): \leftrightarrow \exists x(\operatorname{LForme}(x) \wedge$ nonVForme $(x) \wedge \operatorname{InstSub}(S, x))$

$(\mathrm{D} 21) \operatorname{LA}(S): \leftrightarrow \operatorname{LV}(S) \vee \operatorname{LnonV}(S)$

(D22) $\mathrm{LS}(S): \leftrightarrow \neg \mathrm{LA}(S)$ 


\section{La légitimité historique de la reconstruction proposée}

Dans les passages décisifs de la Wissenschaftslehre Bolzano part, lui aussi, d'une proposition et pose la question de savoir quelles propositions «se laissent développer à partir d'elle » ${ }^{9}$ ou lesquelles «viennent au jour » ${ }^{10}$, lorsqu'on fait varier en elle certaines représentations. De plus, Bolzano introduit immédiatement à la suite de ces passages un concept-clé pour notre présentation, à savoir le concept d'une forme propositionnelle : " on pourrait appeler [les propositions universellement ou pleinement valides] des propositions qui sont vraies d'après leur espèce entière ou d'après leur forme, celles qui sont absolument non valides des propositions qui sont fausses d'après leur espèce entière ou leur forme, attendu que l'on comprend par l'espèce dont on parle ici la collection de toutes les propositions qui ne diffèrent que par les représentations $i, j, \ldots{ }^{11}$.

Ce passage n'est pas, bien entendu, une preuve de la concordance historique de mon analyse. Il montre toutefois quelle est l'importance du concept de forme propositionnelle - compris comme un ensemble de propositions pour Bolzano. Cela n'exclut pas que Bolzano ait pu lui-même défendre l'interprétation courante ou qu'il ait pu lui ouvrir la voie. Mais, le cas échéant, une révision ou une reconstruction "révisée » qui montre la cohérence de l'analyse bolzanienne apparaît d'autant plus importante.

\section{La pertinence de la reconstruction proposée}

(i) L'interprétation courante du concept bolzanien de validité universelle logique est formellement incorrecte et inadéquate dans son contenu. L'analyse que j'ai proposée résout les deux problèmes d'un coup. On pourrait bien sûr sauvegarder l'interprétation courante en abandonnant l'une de ses conditions. C'est la raison pour laquelle j'ai introduit explicitement, malgré le consensus dont elles font l'objet, les présuppositions qui sous-tendent mon analyse.

(ii) Dans les discussions qui portent sur l'analyticité et la validité universelle logique (ou vérité logique) de même que sur la forme logique, le point de départ bolzanien joue souvent un rôle important et l'interprétation

9. Bolzano, 1837, $\$ 147$, vol. 2, p. 82.

10. Bolzano, 1837, $\$ 147$, vol. 2, p. 86 .

11. Bolzano, 1837, $\$ 147$, vol. 2, p. 82 ; Cf. aussi Bolzano, 1837, $\$ 12$, vol. 1 , p. 48 : «Ainsi, la proposition: Certains hommes on la peau blanche, prise individuellement ne figure en logique au mieux que comme exemple, mais certainement pas comme l'objet exclusif d'un principe (Lehrsatz); mais le genre entier de propositions auquel appartient cette dernière apparaît comme un tel objet, à savoir le genre de propositions qu'embrasse l'expression : Certains A sont B. Si on veut maintenant appeler de tels genres de propositions les formes universelles des propositions (malgré que, en fait, seul leur désignation — c'est-à-dire l'expression orale ou écrite de ceuxci, par exemple l'expression : Certains $A$ sont $B$ - devrait être appelée ainsi) : alors on peut dire que la logique ne considère que la forme des propositions, et non les propositions prises individuellement. » 
courante occupe, le plus souvent, le premier rang. Mais, il n'est pas, non plus, rare que des auteurs éminents choisissent des interprétations simplifiées qui recoupent l'interprétation courante de la conception bolzanienne. (On pensera à la formulation de Quine dans «Carnap and Logical Truth» ${ }^{12}$ : "Ainsi, les vérités logiques sont les énoncés vrais qui n'impliquent essentiellement que les mots logiques. ») On ne peut apprécier sérieusement et peser les avantages, les inconvénients de même que les arguments qui parlent en faveur ou en défaveur de la conception classique de la vérité logique et de la validité universelle logique que si on soumet leur point de départ à une discussion adéquate. (En tant que simplifications, de telles interprétations ne présentent bien entendu aucun problème; elles ne deviennent pernicieuses qu'en ceci qu'elles sont répétées machinalement et sans distance critique par plusieurs.) C'est ainsi que la mise au point de l'interprétation courante acquiert une pertinence plus immédiate que la question purement historique de l'exégèse bolzanienne.

(iii) On peut exprimer métaphoriquement la différence entre l'interprétation courante et la conception révisée de la forme logique d'une proposition comme suit : tandis que, d'après l'interprétation courante, ce qui compte en première ligne est la forme qu'on doit «extraire ", d'après la version révisée, ce qui compte est bien plutôt la forme propositionnelle dans laquelle une proposition s'inscrit ou dans laquelle elle s' «insère ». Dans le premier cas, il s'agit d'ensembles de propositions qui peuvent être générés par variation uniforme et simultanée de représentations, dans le second, il s'agit d'ensembles de propositions qui sont la forme, au sens établi plus haut, de propositions quelconques et dont la proposition donnée est un élément ou une instance substitutionnelle. Cela conduit à une révision décisive de ce qu'on retrouve dans les manuels et dans la littérature spécialisée, sous le thème «forme logique».

(iv) D'après la version standard contemporaine, un énoncé est logiquement universellement valide (ou logiquement vrai) si et seulement s'il est vrai sous toutes les interprétations de ses constantes non logiques. Selon la méthode bolzanienne de la variation, les composantes primitives d'une proposition sont échangées pour des représentations arbitraires du domaine de variation correspondant et il s'agit de déterminer si les propositions qui sont générées de la sorte sont vraies ou fausses; selon la méthode courante les composantes primitives non logiques d'un énoncé sont subsumées à un domaine d'entités fixé à l'avance et il s'agit de déterminer si, eu égard à cette subsomption, l'énoncé est vrai ou faux. Lorsqu'on applique la méthode contemporaine, comme c'est souvent le

12. Quine, 1960, p. 352. 
cas, à des exemples du langage ordinaire, le problème technique lié à la méthode substitutionnelle de Bolzano resurgit. (Ce problème disparaît bien entendu d'un seul coup aussitôt qu'on se rapporte à un langage clairement structuré avec un vocabulaire primitif explicite et les règles de formation propositionnelle usuelles.) Par contre, la méthode usuelle ne conduit pas au problème de l'impropriété matérielle, ou du moins n'y conduit pas de la même manière que la méthode substitutionnelle de Bolzano, et ce pour la raison suivante : Une interprétation $I=\langle D, V\rangle$ consiste dans le cas le plus simple en un domaine d'objets $D$ et une fonction d'interprétation $V$; de la sorte, les composantes primitives non logiques d'une proposition sont subordonnées à des entités qui dépendent de $D$, à savoir, les noms singuliers avec des éléments de $D$, et les prédicats n-adiques avec des ensembles de n-tuples d'éléments de $D$. Même lorsqu'un énoncé (ou par exemple une formule du calcul des prédicats) n'est composé que de composantes logiques et ne contient aucune expression non logique, il peut être vrai selon une certaine interprétation et faux sous une autre, tout dépendant du choix du domaine d'objets de D. Pour Bolzano, la représentation la plus générale [quelque chose] reste non-substituable; si on la considérait substituable et la variait, cette variation serait indépendante de la variation des autres composantes de la proposition en soi.

(v) Ce qui a été dit ici à proposition du concept d'analyticité logique vaut bien entendu - mutatis mutandis - également pour tous les autres concepts logiques de Bolzano et en particulier pour son concept de déductibilité logique.

\section{Remarques finales}

Une bonne partie - pour ne pas dire : la plus grande — de ce qui a été écrit à propos des réalisations de Bolzano dans le domaine de la logique reste en deçà de ce que Bolzano a lui-même écrit. Bien peu de choses écrites sur les idées logiques de Bolzano contribuent effectivement à leur éclaircissement et à leur approfondissement. Les simplifications et les vulgarisations ont, bien entendu, leur importance - il n'y a pas lieu de contester cela. Mais pour les fins d'une explication scientifique on ne doit pas s'orienter sur de telles interprétations ou même encore les aplatir davantage qu'elles ne le sont. On doit bien plutôt retourner aux sources originales bolzaniennes et à leurs interprètes classiques, tels Heinrich Scholz et Jan Berg.

(Traduit de l'allemand par Sandra Lapointe) 